\title{
DESIGNING DYNAMIC WEB PAGES IN THE WYSIWYG INTERFACE
}

\author{
David Wolber, Yingfeng Su, Yih-Tsung Chiang \\ Department of Computer Science, University of San Francisco \\ wolber,suyi2,chiayi2@usfca.edu
}

\begin{abstract}
WebSheets is a programming in the WYSIWYG interface tool for building dynamic web pages that connect to databases. The system allows designers to "program" by entering query by example (QBE) and spreadsheet formulas into visual components of HTML documents. The system then automatically generates dynamic web pages that can be executed in a browser.
\end{abstract}

Key words: spreadsheet, programming by example, QBE, dynamic web page

\section{INTRODUCTION}

The web is becoming more dynamic in that more and more pages display dynamic content, computed from live database data, instead of static text and graphics. Unfortunately, our end-user web development tools, such as Front Page (Microsoft, 2002), only allow a designer to create static web pages. Designers that do not program are excluded from creating dynamic pages without the help of a programmer.

Emerging tools such as XMLC (Lutris, 2002) and WebMacro (WebMacro, 2002) do allow the designer to specify the presentation of a dynamic web page. With these tools, the designer uses a WYSIWYG editor to design the layout of the page, entering sample data where the dynamic content will be. The designer then marks these dynamic hotspots in the HTML code. The programmer, working separately, writes code that replaces the hotspots with data computed from a live database data. 
Though template tools allow designers to play a role in dynamic web page creation, designers still cannot build a complete dynamic web page without the aid of a programmer. Such exclusion from the creative process is especially frustrating to designers savvy enough to use spreadsheets and database management systems. These designers possess all the skills necessary to specify all the elements of many dynamic pages (HTML, formulas, database information), but there are no tools that integrate these elements, so the savvy designer's skills are of no help.

We have designed a prototype of such an integrated system, which we call WebSheets. Our approach, called programming in the WYSIWYG interface, is to combine some well-known end-user programming methods, namely spreadsheet formulas, QBE (Zloof, 1977) and Programming by Example (PBE), with a WYSIWYG HTML editor. The dynamic content of a page is specified not with abstract programming or SQL statements, but within the context of the graphical interface (e.g., in table cells). The system then performs the job of the programmer by automatically generating an HTML page and Java servlet based on the designer's specifications.

We have observed a number of designers and experience programmers using the prototype. Our observations suggest that the tool can be used effectively by designers who are not programmers. The feedback we received also suggests that the tool can significantly reduce development time for designers and programmers alike.

While future papers will report on formal usability tests, this paper provides an overview of our approach, including the development process and the code generation. We also compare our approach to those used in Macromedia's Ultradev 4 (Macromedia, 2002), in schema-based, one-click web development systems, and in PBE systems.

\section{THE DEVELOPMENT PROCESS}

A WebSheets designer begins development by entering text, graphics, and components (e.g., tables) just as if a static page were being developed. The difference comes when the designer right clicks on a visual table in order to map it to a database table.

If a designer wishes to map the visual table to an existing database, a dialog is provided that allows the designer to select the database, table, and the columns to map. If no database exists, the designer can enter a sample row in the visual table, and specify that WebSheets automatically generate a database table. Thus, without knowing anything about database tables or columns, a user can create a dynamic web page and its underlying persistent data. 


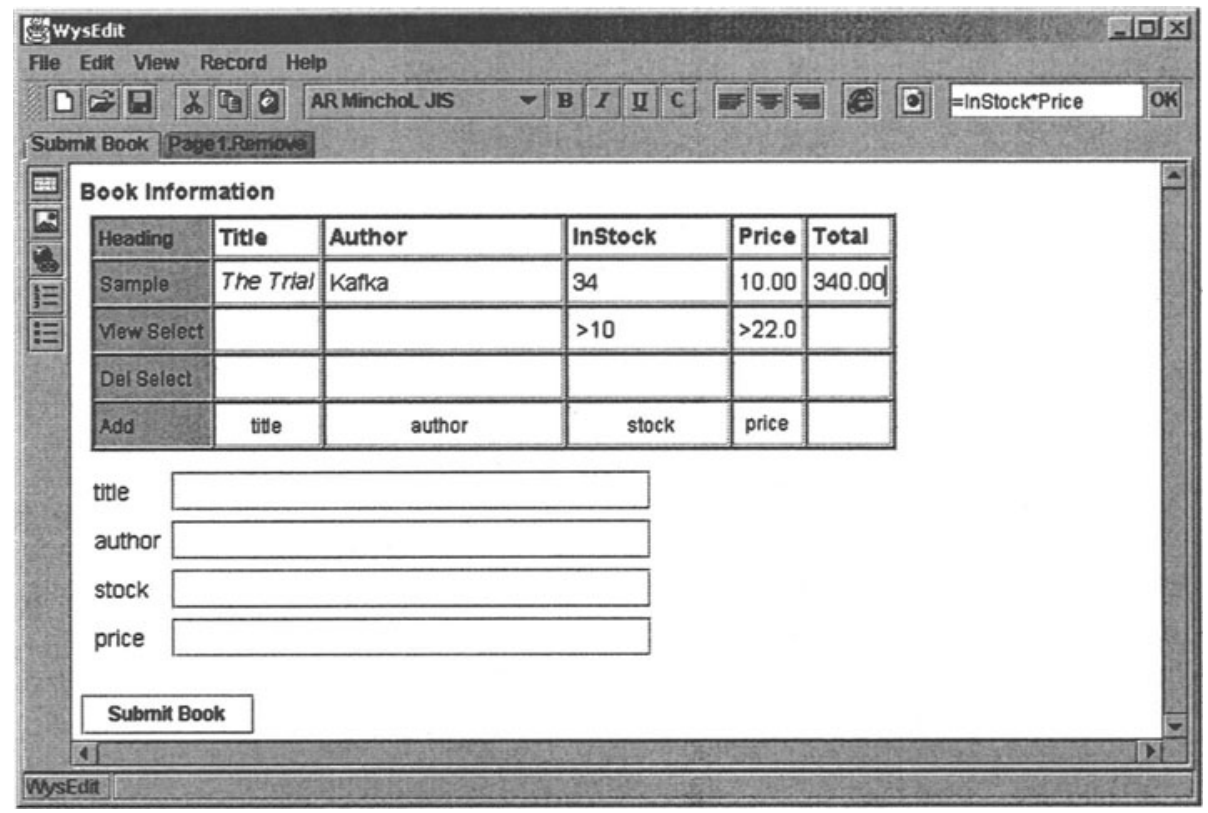

Figure 1. WebSheets Development of a Bookstore page

After the mapping step, the development-time visual table represents both a run-time view and the mapped database table. If the designer runs the dynamic page immediately after the mapping, the resultant web page will display all records of the mapped database table in the visual table. If such a simple web page was required, the designer could just as well have used a one-click web front-end system like ASPapp (ASPapp, 2002) WebSheets focus, however, is to allow designers to specify more complicated dynamic pages.

To facilitate this, a mapped visual table appears with one sample row of "live" database data, and four other auxiliary rows, empty except for labels in the left-most column that help guide the designer (see Figure 1).

\subsection{PBE Formatting and Formulas}

The designer specifies the formatting of all table data by example, that is, by formatting the data in the row labelled "Sample". For instance, in Figure 1, the designer has italicized the title The Trial. When the resulting dynamic page is run in a browser, the titles of all rows will appear italicized.

During the mapping phase, the designer can leave some columns of the visual table unmapped (not corresponding to a database column). The designer can enter spreadsheet-like formulas in these columns. For instance, 
in Figure 1, the designer has entered the formula "=InStock*Price" in the non-mapped column labelled "Total". On entry, formulas are evaluated using the live database data in the other columns of the sample row. This is done only to provide immediate feedback to the user. The computed value (e.g., 340.00) is placed in the cell and the formula appears in the toolbar (as it is in Excel and most spreadsheets).

Just as with the formatting, the formula specified for the sample row is applied to all rows that appear when the resulting dynamic page is run in a browser.

\subsection{Specifying Selection with QBE}

The designer enters QBE expressions in the row labelled "View Select" to specify the database rows that should be displayed. The advantage of QBE is that the designer can specify complex selection without understanding SQL syntax, as all expressions are entered in the context of table cells. For example, suppose the designer enters expressions in the view select row as in Figure 1. The system automatically builds the query:

\section{SELECT * from BookTable where InStock $>10$ and Price $>22.0$}

In the resultant dynamic web page, only the selected books will be displayed.

The row labelled "Del Select" is similar to the "View Select" row, but the QBE entered is used to choose which rows are deleted from the database when the page is invoked. If "Kafka" were entered in the Author column of the "Delete Select" row in Figure 1, the following SQL statement would be generated:

DELETE * from BookTable where Author=Kafka

If the "Del Select" row is left blank, no deletions will occur.

\subsection{Input Forms}

The row labelled "Add" facilitates the specification of input pages in which the end-user enters new records in a table. If the designer enters constant data in this row, a record containing that constant data is entered in the database table each time the page is invoked.

Of course, database operations are often based on something other than constant data. In many dynamic pages, the operations will depend on data entered by the user in an input form. 


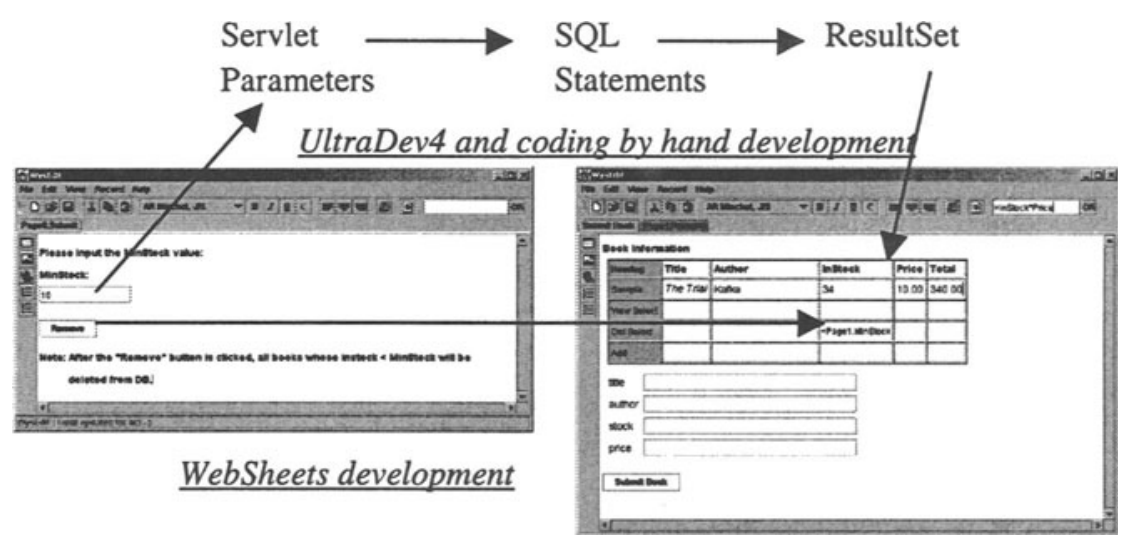

Figure 2. An input form page (left) and a result page (right).

WebSheets allows QBE expressions and spreadsheet formulas to include references to the names of input form data. For instance, in the "Add" row of Figure 1, the designer has entered the names of the input form text boxes (note that the default name of a text component is taken from its label).

At run-time, when the Submit Book button is selected, the values in the text boxes will be used to add a record to the database. After the add operation, the visual table will then be updated using the criteria in the view select row (InStock $>10$ and Price $>22.00$ ).

Formulas and expressions can also refer to input form data from another page in the development environment. The designer just qualifies the component name with the page name, e.g., "page1.MinStock"(See Figure 2).

\subsection{Entry Points}

A dynamic web page can be invoked from submit actions (e.g., buttons) in other pages as well as itself. For instance, the dynamic page in Figure 1 invokes itself (redraws itself with one new record) when the user selects the Submit Book button. It is also invoked by the Remove button on another page, as shown in Figure 2.

A WebSheets designer maps a button to a page by right-clicking on the button and specifying the name of any open page in the development environment. When a page is specified as a result page of a button, a new tab appears in the result page's development-time window. In the right side page shown in Figure 2, there are two tabs-the one corresponding to the "Submit Book" button in the same page, and the selected "Page1.Remove" tab corresponding to the "Remove:" button in the left-side input page. The designer chooses a tab to specify the actions that should occur when the page 
is loaded as a result of clicking the button the tab represents. In Figure 1, the designer has chosen the "Submit Book" tab to specify the actions that should occur when the "Submit Book" button is clicked. In Figure 2, the designer has chosen the "Page1.Remove" tab to specify the actions (e.g., a delete) that should occur when Pagel's "Remove" button is clicked.

\subsection{Code Generation}

After completing the specifications, the designer invokes the code generation operation and web pages are created for each editor page open in the development environment. The pages are automatically registered with a server (currently, a WebLogic (BEA 2002) server), so the designer can "run" the pages and immediately see the results in a browser.

Figure 3 illustrates the WebSheets code generation process. From the designer's WYSIWYG specifications, WebSheets generates an HTML template file suitable for use with the Lutris Ehydra XMLC compiler (Lutris 2002). The HTML template contains id tags for all visual componentseither the default ids WebSheets assigns when the component is inserted, or the ids the designer has explicitly specified. If this template file were sent directly to a browser, all tables would appear with the headers and the single sample row but without the guide rows (View Select row, etc.).

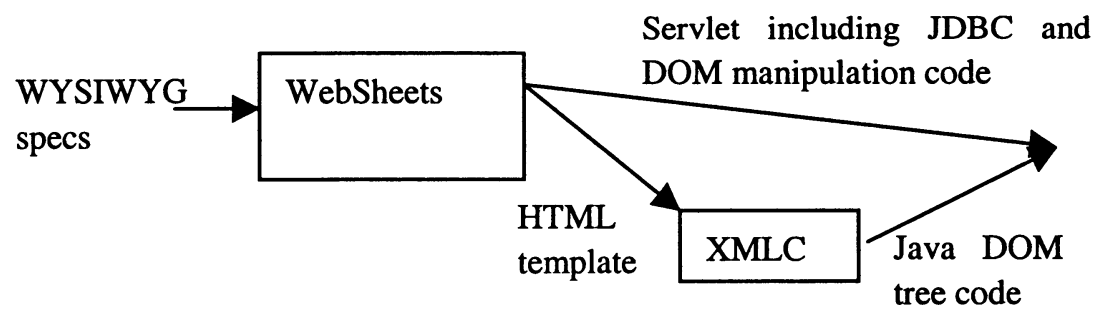

Figure 3. Code Generation in WebSheets

After generating the template file, WebSheets invokes the XMLC compiler. XMLC takes the template HTML file as input and generates Java code representing the page as a Document Object Model (DOM) tree. If this DOM tree were invoked from a servlet, it, too, would display the page with the single sample row specified during development.

Finally, WebSheets generates a Java servlet that manipulates the DOM tree to insert dynamic content. The generated servlet executes the database operations specified in the auxiliary rows of the development-time table, 
then modifies the DOM tree. This servlet is the key automation of the system, essentially replacing the work that generally requires a programmer.

Following is code from the servlet generated from the Bookstore page of Figure 2:

Class BookStoreServlet extends HTTPServlet

$\{1 \ldots$

public void service(HttpServletRequest req, HttpServletResponse res)

throws ServletException, IOException \{

If(actionTag.equals("Page1.Remove")) \{

// Access request parameters

String minStock = req.getParameter("MinStock");

// Access DOM tree created from HTML templ

BookListDOM doc = new BookListDOM();

// Execute specified delete operations using JDBC and SQL Delete statements

stmt.execute("delete from BOOKS where INSTOCK<" + minStock);

// Execute the specified Select statement to obtain a resultset.

rs = stmt.executeQuery (

"select BOOKS.ISBN, BOOKS.NAME,

BOOKS.PRICE, BOOKS.INSTOCK from BOOKS );

// Use DOM manipulation code to:

while(rs.next()) \{

// Use DOM manipulation code to enter these values in the DOM tree.

doc.setText_table1_col0(rs.getString(1));

doc.setText_table1_col1(rs.getString(2));

doc.setText_table1_col2(rs.getString(3));

doc.setText_table1_col3(rs.getString(4));

// Evaluate the spreadsheet formulas of the "expressions"

doc.setText_table1_col4(String.valueOf(rs.getInt(3) * rs.getInt(4)));

// Clone the sample row

tempRow.getParentNode().insertBefore(tempRow.cloneNode(true), tempRow);

\}

// Remove the sample row

tempRow.getParentNode().removeChild(tempRow);

// Write the updated DOM tree to the browser

out.println(doc.toDocument());

\} else if(actionTag.equals(“Submit Book")) \{

II ...

\}

Code Listing 1. Generated Servlet Code 
Since the designer can specify entry points to a page from various input forms, the generated servlet must handle requests from each of those entry points, each of which may send a different set of request parameters. Thus, the code generator associates a particular action tag with each entry point and inserts the tag in the submit action statement of the invoking servlet. The service method of the invoked servlet (which may be the same as the invoking servlet) checks the action tag value and proceeds dependent on it. In the code of Listing1, two action tags have been specified, one for the button named "Page1.Remove", and one for a button named "Submit Book".

The code for each action tag corresponds to the expressions entered in the corresponding tab in the development time environment. If expressions were entered in the "Add" row, the code will contain SQL INSERT statements (wrapped in JDBC code). If expressions were entered in the select delete row, the code will contain SQL DELETE statements (as in Code Listing 1). The code will always contain a SELECT statement based on the expressions entered in the view select row. If no such expressions exist, the generated SELECT statement will not contain any WHERE clauses (this is the situation in Code Listing 1)

After obtaining a result set of live database data from the SELECT statement, the generated code manipulates the DOM tree to insert the data into the HTML table. The code first clones the sample row of the DOM tree in order to access its formatting. It then adds a row in the DOM tree for each of the rows in the result set, setting the data's formatting as specified in the sample row. As it loops through the rows, the code also evaluates any spreadsheet formulas in expression columns and inserts this dynamic data in the DOM tree node for that column. When all rows have been inserted, the code removes the sample row and writes the updated DOM tree to the browser.

\section{RELATED WORK}

This section compares the WebSheets approach to those used in related commercial and research systems.

\subsection{Commercial Web Development}

Macromedia's UltraDev 4 (Macromedia, 2002), the union of Dreamweaver and Cold Fusion, is the commercial system most similar in goals and approach to WebSheets. Like WebSheets, an UltraDev 4 designer can build some dynamic web pages without programming. The systems differ in that UltraDev 4 is more of a visual interface to a program, with 
many dialog boxes and property sheets, whereas WebSheets is more of a programming in the interface system, like a spreadsheet.

Take, for instance, the development of a simple page that allows an enduser to add a record using input form data. In UltraDev 4, the designer creates a ResultSet object that is mapped to the database table. The designer then maps the columns of this ResultSet to columns of the visual table. Next, the UltraDev4 developer chooses the Submit button and maps it to an Add Record operation by choosing it from a list of operations. He then maps the input form's text boxes to the parameters of the page being built. Finally, he maps the page parameters to the parameters of the Add Record operation created earlier. Though performed in a visual manner, the process is similar to the steps a programmer would complete if coding by hand (see the top of Figure 2).

Because database operations are specified within the user interface (e.g., in the "Add" row of a table) the WebSheets designer can avoid thinking in terms of result sets, page parameters, and other programming concepts. The circuitous route of specification, from input form to page parameters to result set to visual table, is also avoided, as the designer can directly specify a mapping between form data and an add record operation by referring directly to the form data in the "Add" row of the table (see the direct link near the bottom of Figure 2).

\subsection{Schema-based Code Generators}

ASPapp (ASPAPP 2002) is indicative of tools that generate dynamic web pages directly from a database schema. These tools provide an immediate (one-click) web front-end to any existing database. Most provide wizards that allow some specialization of the generated pages. A designer can also edit the generated code after the fact to make layout and other modifications, using whatever tools and editors are available.

Since specialization is facilitated through a wizard in schema-based generation, row selection expressions must be specified outside the context of the interface, with SQL-like statements entered in dialogs. In WebSheets, expressions are specified in the context (a table cell) of the target interface.

WebSheets is also designed so that any component in the interface, e.g., a list box, text area, even raw text, can be mapped to database table columns. The wizard approach of a schema-based system can offer flexibility, but the designer must imagine the user interface components when specifying the system, as opposed to beginning with the interface and mapping it to the persistent data. 


\subsection{Declarative Definition of Web Sites}

Similar to schema-based generators are systems such as Strudel (Fernandez, 1998) that provide an intermediate layer between the raw database and the web site. These systems allow a designer to specify a query that describes the structure of the website to be built. From the query, the system builds a data model, often in XML, which represents the part of the database that the website covers. Various methods are then used to map this model to the actual layout of the web pages.

\subsection{Query by Templates}

Query by Templates (QBT) (Sengupta, 1997) is a generalization of QBE. Its focus is visual specification of interfaces for XML data instead of database data. The semi-structured nature of XML data leads to a more natural mapping of non-tabular graphical components to the data, so QBT users can specify queries within "components" such as poems.

\subsection{Forms/3 Spreadsheets}

Forms/3 (Burnett, 2001) is a programming environment based on the spreadsheet. Spreadsheet formulas need not be placed only in cells of a table, but can be entered in any visual component or even as a property of some complex component such as a graphic. Tables need not be of a fixed size, but can contain a variable number of rows and columns.

Like with regular spreadsheets, the data entered in a Forms $/ 3$ interface is persistent. But though there is the concept of dynamically sized tables, there is no notion of an underlying store beneath the visible data that the designer manipulates. Thus, it cannot be used to create interfaces to existing databases or to specify traditional database operations.

\subsection{Programming by Example}

PBE systems are intelligent macro facilities. Instead of just recording a designer's demonstrations and later playing them back, these systems infer a generalized program. PBE has been applied successfully in many domains, the results of which have been reported in two books (Cypher et. al., 1993, Lieberman, 2001). It has even been applied to dynamic web page development, but only in the context of building wrapper programs (Kushmerick, 2000, Bauer et. al., 2001), i.e., pages that get data from other already existing web pages. 
Led by the first author's previous experience (Wolber, et. al. 2001, Myers et al, 2000), our original idea was to apply PBE to the development of web pages that connect to databases. The system would display the entire "live" database table at development time, instead of a single sample row, thereby making the system more WYSIWYG. Instead of specifying formulas and $\mathrm{QBE}$, as in WebSheets, the designer would demonstrate examples of the database operations using sample data. To specify an Add Record operation, for instance, the designer would add a row to the visual table, then cut and paste data from the input form to the new row. To specify selection criteria, the designer would provide sample rows that fit the criteria, and the system would infer the SQL Select statement. In this manner, abstract variable names would be unnecessary because the designer would work only with sample data.

But after analyzing paper and partial prototypes, we came to the conclusion that the concreteness afforded by PBE was not worth it for our target domain (dynamic web pages that access databases). Users found the manipulations to demonstrate an Add Record operation laborious, compared to just providing an auxiliary add row and allowing the designer to specify what to add in a spreadsheet manner (even if variable names had to be used). Delete and select were also found problematic, as numerous examples were necessary for the system to infer a generalized selection expression. Finally, providing feedback and editing of an inferred program is notorious difficult for PBE systems. With our spreadsheet-like approach, this problem was solved, since the designer can always see the "program" specification directly in the WYSIWYG interface.

For these reasons, we chose to abandon PBE as the basis for WebSheets. In the current prototype, PBE is used only in the sense that formatting and formulas are specified in the sample row and then automatically applied to all rows, and a user can provide a sample row and the system will generate a database table.

\section{STATUS AND FUTURE WORK}

WebSheets is a research prototype based on a rudimentary HTML editor. The table widget only allows conjunction (AND) expressions to be specified, and spreadsheet formulas are limited to mathematical operators and a small built-in function set. These limitations can easily be fixed. For instance, we are currently implementing a COM connection that will allow any Excel formula to be specified.

The prototype has been completed to the point that our basic approach can be tested. It has been used to create complete web applications such as 
the Bookstore example illustrated in this paper. Feedback from users has been encouraging-most are amazed at how quickly dynamic web pages can be built.

We have begun to perform extensive usability testing in order to refine the system and verify its usefulness. We also plan to make a more complete WebSheets version publicly available so as to get widespread feedback.

In the next version, we will implement dynamic visual components other than tables. A designer will be able to "program" lists, combo boxes, and sets of text cells in the same manner as tables. We will also design and implement a method for specifying updates to table rows, a capability not covered in the current system.

One direction of future work concerns the specification of relational tables in the WebSheets framework. Currently, a database table will be created from a sample row, but no relations are inferred. We are currently exploring a number of techniques for specifying relations in the WYSIWYG interface, including inferring such relations from multiple sample rows and from analyzing the links between pages.

Following the lead of (Sengupta, 1997), we would also like to explore mapping the designer's visual specifications to XML data, instead of databases, and generating XML data, instead of a table, from sample row(s).

\section{CONCLUSION}

WebSheets is not completely programming in the WYSIWYG. In fact, it is based on a table widget with auxiliary rows that are seen only during development, and not at run-time. However, the auxiliary rows allow database operations (select, add, delete) and formulas to be specified in the context of the user interface, that is, in the column-based manner that has long been effective in Spreadsheets and QBE systems.

WebSheets combines this in-context specification with a method for defining interaction, that is, the way a dynamic page should change dependent on how it is invoked. By representing the various invocations of a page with a tabbed window, the designer can separately specify the operations that should occur when the user clicks on, for example, an "Add Record" button and a "View Records" button. Our goal is to refine this basic approach to the point that designers can build complete, customizable and complex dynamic web pages. 


\section{REFERENCES}

ASPapp,Inc. (2002), http://www.aspapp.com

Bauer, M. Dengler, D., Paul, G., and Meyer, M.(2001) Programming by Demonstration for Information Agents, chapter in Your Wish is my Command, Henry Lieberman, Ed. San Francisco: Morgan Kaufmann, 2001

BEA, Inc. (2002)., http://www.bea.com.

Burnett, M. Atwood, J., Djang, R. , Reichwein, J., Gottfried, H. and Yang,S.(2001),Forms/3: A First-order Visual Language to Explore the Boundaries of the Spreadsheet Paradigm, Journal of Functional Programming, 11(2):155-206, Cambridge University Press.

Cypher, A. (1993) Watch What I do: Programming By Demonstration, MIT Press.

Fernandez, M., Florescu, D, Kang, J., Levy, A., and Suciu, D. (1998) Catching the Boat with Strudel: Experiences with a Web-site Management System, in Proc. of ACM SIGMOD Conference on Management of Data, Seattle, WA. ,ACM.

Florescu, D., Levy, A., and Mendelzon, A.,(1998)Database Techniques for the World-Wide Web: A Survey, SIGMOD Record, 27(3):59-74, ACM..

Kushmerick, N. (2000) Wrapper Induction: Efficiency and Expressiveness, Artificial Intelligence, 118, 2000, pp. 15-66.

Lieberman, H. ed. (2001) Your Wish is My Command: Programming by Example, MorganKaufmann.

Lutris, Inc. (2002), http://www.lutris.com/products/index.html

Macromedia (2002), http://www.macromedia.com

Miller, R., Myers, B., (1997), Creating Dynamic World Wide Web Pages by Demonstration. Carnegie Mellon University School of Computer Science Technical Report, no. CMU-CS97-131 and Human Computer Interaction Institute Technical Report, CMU-HCII-97-101.

Microsoft, Inc. (2002), http://www.microsoft.com

Myers, B., McDaniel R., and Wolber, D.,(2000), Intelligence in Demonstrational Interfaces, in Communications of the ACM, March, 2000. vol. 43, no. 3. pp. 82-89, ACM.

Sengupta, A. Dillon, A., (1997), Query by Templates: A Generalized Approach for visual query formulation for text dominated databases. In the Conference on Advanced Digital Libraries (ADL '97).

WebMacro (2002), http://www.webmacro.org

Wolber, D., and Myers, B. (2001), "Stimulus-Response PBD: Demonstrating When as Well as What", Your Wish is My Command, Lieberman, H. ed. San Francisco: Morgan Kaufmann.

Zloof, M., (1977),"Query-by-Example: A Database Language", IBM Systems J., 21(3), IBM. 


\section{BIOGRAPHIES}

David Wolber is an associate professor in the University of San Francisco's department of computer science. His research interests include recommender systems, programming by demonstration, and visual programming.

Yingfeng Su is completing a Master's degree in computer science at the University of San Francisco.

Yih-Tsung Chiang is completing a Master's degree in computer science at the University of San Francisco. 